\title{
Hypothesis: Pentoxifylline is a potential cytokine modulator therapeutic in COVID-19 patients
}

\author{
Bruce Hendry ${ }^{1}$, NIna Stafford ${ }^{2}$, Ahran Arnold $^{3}$, Arvind Sangwaiya $^{2}$, Vijay Manglam $^{2}$, \\ Stuart Rosen ${ }^{3}$, and Jayantha Arnold ${ }^{2}$ \\ ${ }^{1}$ Kings College London \\ ${ }^{2}$ London North West University Healthcare NHS Trust \\ ${ }^{3}$ National Heart and Lung Institute
}

June 15,2020

\begin{abstract}
We propose a new hypothesis that the established drug pentoxifylline deserves attention as a potential repurposed therapeutic for COVID-19. Pentoxifylline is an anti-inflammatory agent that suppresses adenosine responses, reduces Tumour Necrosis Factor alpha, Interleukin 1, Interleukin 6 and Interferon gamma and may act to reduce tissue damage during the cytokine storm response to SARS-CoV-2 infection. This agent has been used clinically for many years and has a favourable profile of safety and tolerability. Pre-clinical data support pentoxifylline as effective in cytokine-driven lung damage. Clinical studies of pentoxifylline in radiation and cytokine-induced lung damage in humans are positive and consistent with anti-inflammatory efficacy. Pentoxifylline is a readily available, off-patent, inexpensive drug suitable for large scale use, including in resourcelimited countries. Current trials of therapeutics are largely focussed on the inhibition of viral processes. We advocate urgent randomised trials of pentoxifylline for COVID-19 as a complementary approach to target the host responses.
\end{abstract}

Hypothesis: Pentoxifylline is a potential cytokine modulator therapeutic in COVID-19 patients Prof Bruce M Hendry*

Dr Nina Stafford **

Dr Ahran D Arnold ***

Dr Arvind Sangwaiya **

Dr Vijay Manglam **

Prof Stuart D Rosen ***

Prof Jayantha Arnold **

* Renal Sciences, Department of Inflammation Biology, King's College London, London SE5 9RJ, UK

** Department of Gastroenterology, Ealing Hospital, London North West University Healthcare NHS Trust, Uxbridge Road, Southall, UB1 3HW, UK.

*** National Heart and Lung Institute, Imperial College London, London W12 0HS.UK.

Corresponding Author: Professor Bruce Hendry KCL, 10 Cutcombe Road, London, SE5 9RJ, UK

Email: bruce.hendry@kcl.ac.uk

Telephone: +447973456694 


\section{Summary}

We propose a new hypothesis that the established drug pentoxifylline deserves attention as a potential repurposed therapeutic for COVID-19.

Pentoxifylline is an anti-inflammatory agent that suppresses adenosine responses, reduces tumour necrosis factor $\alpha$, interleukin 1, interleukin 6 and interferon $\gamma$ and may act to reduce tissue damage during the cytokine storm response to SARS-CoV-2 infection. This agent has been used clinically for many years and has a favourable profile of safety and tolerability. Pre-clinical data support pentoxifylline as effective in cytokine-driven lung damage. Clinical studies of pentoxifylline in radiation and cytokine-induced lung damage in humans are positive and consistent with anti-inflammatory efficacy.

Pentoxifylline is a readily available, off-patent, inexpensive drug suitable for large scale use, including in resource-limited countries.

Current trials of therapeutics are largely focussed on the inhibition of viral processes. We advocate urgent randomised trials of pentoxifylline for COVID-19 as a complementary approach to target the host responses.

\section{Keywords}

COVID-19, cytokine, immunomodulation, pentoxifylline, pneumonia, SARS-CoV-2, viral

\section{Running title}

\section{Pentoxifylline and COVID-19}

\section{Background}

COVID-19 is a disease caused by the SARS-CoV-2 virus, characterised by an early mild- moderate viral syndrome of fever, tiredness, cough and headache [1]. Over $80 \%$ of patients have a self-limiting illness not requiring hospital admission and show clear improvement in two weeks. A minority of COVID-19 patients progress through a transition phase around days 7-11 of worsening pulmonary complications [1]. These manifest as breathlessness, acute lung injury and respiratory failure, and often progress to require mechanical ventilation with subsequent high mortality. This deterioration appears to be driven by lung host responses including a cytokine storm of inflammation leading to severe tissue damage and irreversible organ failure likened to adult respiratory distress syndrome (ARDS) [2]. Patients who develop ARDS are at very high risk of death.

The cytokine storm phase of COVID-19 is associated with increased production of a range of inflammatory cytokines including interleukin-1 $\beta$ (IL1 $\beta$ ), interleukin-6 (IL6), tumour necrosis factor- $\alpha$ (TNF $\alpha$ ) and interferon- $\gamma(\mathrm{IFN} \gamma)$ [3]. Several case series have reported increased TNF $\alpha$ levels in patients with COVID-19 and particularly high levels appear to be associated with a severe disease course [3,4]. One series has described increased TNF $\alpha$ inducibility in macrophages, in the presence of SARS-CoV-2 virus [4]. TNF $\alpha$, as the master regulator of cytokines, is considered key in both immune pneumonitis and acute myocardial injury witnessed in COVID-19.

Current COVID-19 therapeutic studies are mainly focused on agents designed to target viral processes or virus-host interactions, for example with Remdesivir. We note that Remdesvir has recently been approved by the UK government, after "numerical reduction in time to clinical improvement", but failing to meet statistical significance on the primary clinical endpoint of mortality [5].

An alternative therapeutic approach is to target the host responses that underlie the cytokine storm and associated inflammation [3]. We, and other colleagues [6], have called for randomised clinical trials of antiTNF agents, such as Infliximab, to treat the cytokine storm induced by SARS-CoV-2 - see our BMJ Rapid Response published online on 9 April 2020, available at: https://www.bmj.com/content/369/bmj.m1439/rr. Pentoxifylline is a cytokine-modulating anti-inflammatory agent with many actions that might reasonably be expected to be therapeutic in the transition phase of COVID-19. 


\section{Description of hypothesis}

\section{Molecular and cellular actions of Pentoxifylline}

Pentoxifylline is a xanthine derivative drug with a wide range of actions at the cellular and molecular level. pentoxifylline has rheological actions increasing erythrocyte deformability and was originally licensed for the treatment of peripheral vascular disease on the basis of suggested improvement of microvascular and capillary blood flow [7]. More recently pentoxifylline has been determined to have extensive anti-inflammatory properties [8]. pentoxifylline inhibits 5'-nucleotidase and phosphodiesterases (PDE). PDE inhibition results in increased cAMP levels, increased protein kinase A (PKA) activity and altered transcriptional regulation of pro-inflammatory genes through modulation of the $\mathrm{NF} \varkappa \mathrm{B} / \mathrm{I} \varkappa \mathrm{B}$ pathway [8]. Pentoxifylline downregulates transcription and expression levels of TNF $\alpha$, IL1b, IL6, IFN $\gamma$, ICAM1 and VCAM1. pentoxifylline 5 '-nucleotidase inhibition reduces the production of adenosine and inosine from adenosine monophosphate (AMP) and inosine monophosphate (IMP) respectively. Pentofxyfilline appears able to downregulate the pathologically important pro-inflammatory adenosine receptor A2A pathway [9]. These effects contribute to the extensive actions of pentoxifylline in reducing pro-inflammatory signals. For example, pentoxifylline reduces cytokine release from pulmonary macrophages derived from patients with sarcoidosis [10]. Lungs have the highest proportion of total resident macrophages in the human body, around 1 trillion.

\section{Pentoxifylline therapy in pre-clinical studies}

In rats pentoxifylline downregulates a range of inflammatory cytokines in the context of sepsis and improves lung function [10]. In animal studies pentoxifylline is effective as a therapeutic in a range of models of lung injury including radiation-induced damage, cytotoxic agent damage and aortic clamping [11]. The actions of pentoxifylline in reducing lung damage appear to depend on a reduction of activity of the adenosine receptor A2A [9]. The direct inhibition of 5'- nucleotidase activity by pentoxifylline likely reduces adenosine production and may therefore complement the actions on cytokine gene transcription. Pentoxifylline improves glomerular damage and reduces $\mathrm{TNF} \alpha$ in crescentic glomerulonephritis in rats [12]. Pentoxifylline does not appear to alter the replication of SARS-CoV in mice and does not show direct antiviral activity.

\section{Clinical studies of pentoxifylline}

The anti-inflammatory properties of pentoxifylline have been confirmed in a range of clinical studies in diverse organ failure syndromes. In severe renal disease patients exhibit resistance to erythropoietin through a proinflammatory state characterised by increased $\mathrm{TNF} \alpha, \mathrm{IFN} \gamma$ and IL6. pentoxifylline improves erythropoietin sensitivity in these patients and this action is associated with reductions in serum $\mathrm{TNF} \alpha$ and IFN $\gamma$ [13]. In diabetic nephropathy pentoxifylline reduces proteinuria through inhibition of intraglomerular inflammatory signals. Clinical studies have demonstrated pentoxifylline as effective in reducing lung damage in the context of radiation damage, and cardiopulmonary bypass [13,14]. Ustunsoy et al (2003) for example found that pentoxifylline reduced levels of TNF $\alpha$ and IL6 when given at the time of bypass [14].

One of the largest scale clinical trials of pentoxifylline anti-inflammatory effects within the last decade, was the STOPAH trial [15]. Examining potential benefit in acute alcoholic hepatitis, no survival benefit was demonstrated, however no safety issues were reported in an inherently vulnerable, immunocompromised patient cohort. pentoxifylline preferential inhibition of macrophage function in alveoli, as opposed to hepatocytes, potentially explains this outcome [16].

Rainsford (2006) reviewed possible treatments for the lung complications associated with inflammatory cytokines in H5N1 "bird flu" and suggested that pentoxifylline should be considered for clinical trials in view of its pharmacology and safety profile [17]. These arguments appear equally suited to the case of COVID-19. Although we cannot describe the SARS-CoV-2 pulmonary syndrome as identical to that seen in H5N1, there are certainly parallels.

TNF has a pivotal role in orchestrating the production of a pro-inflammatory cytokine cascade. TNF $\alpha$ is thus considered to be a 'master regulator' of pro-inflammatory cytokine production [18]. Post mortem lung biopsies in COVID-19 showed interstitial oedema which would normally be the result of TNF induced 
increased capillary permeability $[19,20]$. This non cardiogenic pulmonary oedema (both interstitial and intraalveolar) is often the first stage of COVID-19 acute lung injury that progresses through the cytokine storm to ARDS [20]. The recent description of thrombosis and endothelialitis in COVID-19 [20] raises the possibility that the rheological actions of pentoxifylline could have benefit in maintaining microvascular function.

Pentoxifylline has over 50 years safety record data of use in humans and has an extensive evidence base for tolerability and safety. Nevertheless, its safety in the context of COVID-19 has not been established and this would need close monitoring in a clinical trial setting. It is available in oral form with good bioavailability, and also can be delivered by intravenous injection. The usual dose orally is $1.2 \mathrm{~g}$ daily in 3 divided doses. An inhalational formulation has been developed, originally for use in neonates. In COVID-19 the cytokine storm appears to be strongly centred in lung tissue, and accordingly inhaled pentoxifylline could be an optimum method for delivery at the highest concentrations where it is most effective, with minimal systemic exposure.

Use of a repurposed drug for COVID-19 may have multiple advantages in addition to its safety and tolerability experience. It is widely available as a generic agent, with multiple sources of supply and therefore manageable cost. There should be no patent protection issues in redirecting the agent to trials in COVID-19. This should be of acute interest as limited-resource areas begin to amass cases of COVID-19.

\section{Design of studies of pentoxifylline in COVID-19}

Study of pentoxifylline in COVID-19 should be feasible and ethical given the well-described adverse event profile of the drug. A randomised study in COVID-19 patients presenting with, or at high risk of, pulmonary complications could be designed with pentoxifylline versus a placebo or comparator treatment, alongside standard of care. pentoxifylline has been used at 400-1200 mg daily in 400mg doses. Initial study likely should use $1200 \mathrm{mg}$ daily in divided doses. If there is initial evidence of benefit with oral pentoxifylline, then study of inhaled pentoxifylline could be valuable in selected patients. In the future it will also be logical to study the combination of cytokine-modifying therapy with direct anti-viral therapeutics (such as the recently favoured Remdesivir). The efficacy of pentoxifylline can be assessed by randomised control trials with key endpoints including mortality, need for ventilatory support, time on ventilatory support, measures of oxygen exchange efficiency and time in hospital.

\section{Conclusions}

The full cellular, pre-clinical and clinical profile of pentoxifylline suggests that pentoxifylline could be effective in reducing the severity of lung injury in patients with COVID-19. These actions could reduce the need for critical care interventions and reduce the burden and mortality of COVID-19 in selected individuals.

If the theoretical and rheological therapeutic benefit of pentoxifylline for COVID-19 is clinically demonstrable, it may prove to be an inexpensive, and readily available, treatment strategy to target harmful cytokine excess in this disease.

We advocate urgent randomised trials of pentoxifylline for patients infected with SARS CoV-2.

\section{List of Abbreviations}

AMP: adenosine monophosphate ARDS: adult respiratory distress syndrome CRP: C-reactive protein ICAM1: intercellular adhesion molecule 1 IFN $\gamma$ : interferon gamma IkB: Inhibitory factor kappa B IL: interleukin IMP: inosine monophosphate NkB: nuclear factor kappa B PDE: phosphodiesterases PKA: protein kinase A SARS: severe acute respiratory syndrome $\mathrm{TNF} \alpha$ : tumour necrosis factor alpha

VCAM1: vascular cell adhesion molecule 1

\section{Declarations}

None of the authors have any relevant actual or potential conflicts of interest.

BMH has received honoraria for speaking or consulting from AstraZeneca, Gilead Sciences, Otsuka, Retrophin, Sanofi, UCB and ViiV Healthcare 


\section{Contributions}

All authors contributed equally

Ethics Approval Not applicable

Consent for Publication Not applicable

Availability of Data and Materials

Not applicable

Funding Not applicable

\section{Acknowledgements}

Not applicable

\section{References}

1. Wang D, Hu B, Hu C, Zhu F, Liu X, Zhang J et al. Clinical Characteristics of 138 Hospitalized Patients With 2019 Novel Coronavirus-Infected Pneumonia in Wuhan, China. JAMA. 2020; Feb 7. doi: 10.1001/jama.2020.1585.

2. Ye Q, Wang B, Mao J. The pathogenesis and treatment of the 'Cytokine Storm' in COVID-19. J Infect. 2020; Apr 10. pii: S0163-4453(20)30165-1. doi: 10.1016/j.jinf.2020.03.037.

3. Huang C, Wang Y, Li X, Ren L, Zhao J, Hu Y, Zhang L et al. Clinical features of patients infected with 2019 novel coronavirus in Wuhan, China. Lancet Jan 2020; 395: 497-506. Doi: 10.1016/S01406736(20)30183-5

4. Giamarellos-Bourboulis et al., Complex Immune Dysregulation in COVID-19 Patients with Severe Respiratory Failure, Cell Host \& Microbe (2020). doi: 10.1016/j.chom.2020.04.009.

5. Wang Y, Zhang D, Du G, Du R, Zhao J, Jin Y, Fu S, Gao L, Cheng Z, Lu Q, Hu Y. Remdesivir in adults with severe COVID-19: a randomised, double-blind, placebo-controlled, multicentre trial. The Lancet. 2020 Apr 29.

doi.org/10.1016/S0140-6736(20)31022-9

6. Feldman M, Maini RN, Woody JN, Holgate GW, Rowland M et al. Trials of anti-tumour necrosis factor therapy for COVID-19 are urgently needed. Lancet published online on 9 April 2020. Doi: 10.1016/S0140$6736(20) 30858-8$

7. Dettelbach HR, Aviado DM. Clinical pharmacology of pentoxifylline with special reference to its hemorrheologic effect for the treatment of intermittent claudication. J Clin Pharmacol. 1985; 25 :8-26. doi: 10.1002/j.1552-4604.1985.tb02796.x

8. Donate-Correa J, Tagua VG, Ferri C, Martin-Nunez E, Hernandez-Carballo C, Urena-Torres P et al. Pentoxifylline for Renal Protection in Diabetic Kidney Disease. A Model of Old Drugs for New Horizons. J Clin Med. 2019; 8: E287. doi: 10.3390/jcm8030287.

9. Konrad FM, Neudeck G, Vollmer I, Ngamsri KC, Thiel M, Reutershan J. Protective effects of pentoxifylline in pulmonary inflammation are adenosine receptor A2A dependent. FASEB J. 2013; 27:3524-35. doi.org/10.1096/fj.13-228122.

10. Tong Z1, Dai H, Chen B, Abdoh Z, Guzman J, Costabel U. Inhibition of cytokine release from alveolar macrophages in pulmonary sarcoidosis by pentoxifylline: comparison with dexamethasone. Chest. 2003; 124:1526-32. doi: 10.1378/chest.124.4.1526 
11. Sunil VR, Vayas KN, Cervelli JA, Malaviya R, Hall L, Massa CB et al. Pentoxifylline attenuates nitrogen mustard-induced acute lung injury, oxidative stress and inflammation. Exp Mol Pathol. 2014; 97: 89-98. Doi: 10.1016/j.yexmp.2014.05.009

12. Chen YM, Ng YY, Lin SL, Chiang WC, Lan HY, Tsai TJ. Pentoxifylline suppresses renal tumour necrosis factor-alpha and ameliorates experimental crescentic glomerulonephritis in rats. Nephrol Dial Transplant. 2004; 19: 1106-15. Doi: 10.1093/ndt/gfh127

13. Ozturk B, Egehan I, Atavci S, Kitapci M. Pentoxifylline in prevention of radiation- induced lung toxicity in patients with breast and lung cancer: a double-blind randomized trial. Int J Radiat Oncol Biol Phys. 2004; 58: 213-9. Doi: 10.1016/s0360-3016(03)01444-5

14. Ustunsoy H, Sivrikoz MC, Bakir K, Senkaya, Erbagci B, Ucak R et al. The inhibition of pro-inflammatory cytokines with pentoxifylline in the cardiopulmunary bypass lung. Respir Med. 2002; 96: 275-9 https://dnb.info/109817061X/34

15. Thursz M, Richardson P, Allison M et al. Prednisolone or Pentoxifylline for alcoholic hepatitis. N Eng J Med 2015;372:1619-1628. doi:10.1056/NEJMoa1412278.

16. Marques LJ, Zheng L, Poulakis N, Guzman J, Costabel U. Pentoxifylline inhibits TNF-alpha production from human alveolar macrophages. Am J Respir Crit Care Med. 1999;159(2):508-11. doi:10.1164/ajrccm.159.2.9804085.

17. Rainsford KD. Influenza ("Bird Flu"), inflammation and anti-inflammatory/analgesic drugs. Inflammopharmacology. 2006; 14: 2-9. doi: 10.1007/s10787-006-0002-5

18. Parameswaram N and Patial S. Tumor Necrosis Factor- $\alpha$ Signaling in Macrophages. Crit Rev Eukaryot Gene Expr. 2010 March 30;20(2):87-103. Parameswaran N, Patial S. Tumor necrosis factor- $\alpha$ signaling in macrophages. Crit Rev Eukaryot Gene Expr. 2010;20(2):87-103. doi:10.1615/critreveukargeneexpr.v20.i2.10

19. Brauer R, Chen P. Influenza leaves a Trail to pulmonary Oedema. J Clin Invest. 2016;126(4):1245-1247. doi:10.1172/JCI86802

20. Ackermann M, Verleden SE, Kuehnel M et al. Pulmonary Vascular Endothelialitis, Thrombosis, and angiogenesis in COVID-19. NEJM May 21 2020. Doi: 10.1056/NEJMoa2015432 